\title{
Nuclear polarization in hydrogenlike ${ }_{82}^{208} \mathrm{~Pb}^{81+}$
}

\author{
Akihiro Haga $^{1}{ }^{*}$, Yataro Horikawa ${ }^{2}{ }^{\dagger}$ and Yasutoshi Tanaka ${ }^{1}$ \\ ${ }^{1}$ Department of Environmental Technology and Urban Planning, \\ Nagoya Institute of Technology, Gokiso, Nagoya 466-8555, Japan \\ ${ }^{2}$ Department of Physics, Juntendo University, Inba-gun, Chiba 270-1695, Japan
}

(5 October 2001)

\begin{abstract}
We calculate nuclear-polarization energy shifts for hydrogenlike ${ }_{82}^{208} \mathrm{~Pb}^{81+}$. A retarded transverse part as well as the Coulomb part is taken into account as the electromagnetic interaction between an electron and the nucleus. With a finite charge distribution for the nuclear ground state and the random-phase approximation to describe the nuclear excitations, we obtain nuclear polarization energy of the $1 s_{1 / 2}$ state as $-38.2(-37.0) \mathrm{meV}$ in the Feynman (Coulomb) gauge. For the $2 s_{1 / 2}, 2 p_{1 / 2}$ and $2 p_{3 / 2}$ states, they are -6.7 ($6.4),-0.2(-0.2)$ and $+0.0(+0.0) \mathrm{meV}$, respectively. The seagull term in the two-photon exchange diagrams is shown to be quite important to obtain the gauge invariance of nuclear polarization energies.
\end{abstract}

PACS number(s): 31.30.Gs, 31.30.Jv, 12.20.Ds

\section{INTRODUCTION}

High-precision Lambshift measurement on high-Z hydrogenlike atoms 1 arose a renewed interest in the quantum electrodynamic (QED) calculation of electronic atoms. Comparison of theoretical results with corresponding experimental data allows sensitive tests of QED in strong electromagnetic fields [2,3]. Any discrepancy between theory and experiment may either motivate an improvement of theoretical calculations and a refinement of experiments, or it may indicate a possible influence of non-QED effects. In this context, the study of nuclear polarization (NP) contributions to the total energy shift of atomic levels becomes important because as a background effect, it represents a natural limitation of any highprecision test of QED. Unfortunately, evaluation of NP is not practicable on the first principle. Any calculation of NP is inherently phenomenological and depends on the parameters of the nuclear model used to describe the intrinsic nuclear dynamics.

\footnotetext{
*Electronic address: haga@npl4.kyy.nitech.ac.jp

${ }^{\dagger}$ Electronic address: horikawa@sakura.juntendo.ac.jp
} 
During the past years, a lot of experience has been accumulated in calculating the NP effect for muonic

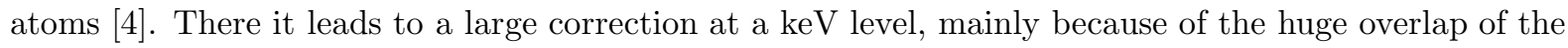
muon-wave function with a nucleus and because the transition energies in muonic atoms are of the order of magnitude of typical nuclear excitation energies.

Much less attention has been paid to the NP effect for electronic atoms. They turn out to be reduced by orders of magnitude because of the small overlap of the electron-wave function with the nucleus and because the transition energies in electronic atoms are in general orders of magnitude smaller than typical nuclear excitation energies.

The NP effect for electronic atoms was first calculated in terms of the second-order Schrödinger perturbation theory [5]. A relativistic field-theoretical treatment of nuclear polarization calculation was then presented by Plunien et al. [6 8] utilizing the concept of effective photon propagators with nuclear polarization insertions. The formalism allows to take into account the effect of the electron negative-energy intermediate states besides the usual contribution of the electron excited into higher unoccupied intermediate states. They found that in electronic atoms NP energies become small due to the cancellation between contributions of positive energy states and those of negative energy states.

In the above studies, only the Coulomb interaction was considered based on the argument that the relative magnitude of transverse interaction is of the order of $(v / c)^{2}$ and the velocity $v$ associated with nuclear dynamics is mainly nonrelativistic. However, it may not be justified. In fact the importance of the transverse interaction has been reported for muonic atoms [9, 10]. In Ref. [10], the transverse nuclear polarization has been studied in order to explain the discrepancies between theory and experiment in the $2 \mathrm{p}$ and $3 \mathrm{p}$ fine-structure splitting energies of muonic ${ }_{82}^{208} \mathrm{~Pb}$. The contribution for the muonic $1 s_{1 / 2}$ state amounts to $20 \%$ of that of the Coulomb interaction.

The transverse interaction is expected to be much more important for electronic atoms than for muonic atoms because of its long-range nature. The transverse nuclear polarization for heavy electronic atoms was first studied by Yamanaka et al. [11,12] using the Feynman gauge and a collective model for the nuclear excitations. They found that the transverse contribution is several times larger than the Coulomb contribution in heavy electronic atoms before the contributions of the positive and negative energy states cancel. However, due to nearly complete cancellation between them, the total NP energy becomes very small.

The purpose of the present paper is twofold; The one is to see how much NP energies are expected for a practically best available model of the ${ }_{82}^{208} \mathrm{~Pb}$ nucleus. For this purpose the Dirac-electron wave 
functions are solved in the Coulomb potential with a finite nuclear charge distribution and the randomphase approximation (RPA) is used to describe the nuclear excitations. The other is to see whether NP energies are sensitive to the choice of the gauge. For this purpose NP energies are calculated both in the Feynman and Coulomb gauges. We will see that NP calculation with only the ladder and cross diagrams shows large gauge dependence and inclusion of the seagull diagram removes most of its gauge dependence 13,14].

Calculations are carried out in momentum space. They involve only double integrals, which are easily carried out with high precision.

\section{NUCLEAR POLARIZATION CALCULATION}

The second-order contributions to the nuclear polarization are given by three Feynman diagrams in Fig. 1. (Here we regard the seagull graph as one of the nuclear polarization diagrams.) Two photons are exchanged between a bound electron and a nucleus and the nuclear vertices are understood to have no diagonal matrix elements for the ladder and cross diagrams, and no nuclear intermediate states for the seagull diagram.

The nuclear-polarization energy shift due to the ladder and cross diagrams is given by [6],

$$
\begin{aligned}
\Delta E_{N P}=i(4 \pi \alpha)^{2} \int d^{4} x_{1} \cdots d^{4} x_{4} \bar{\psi}\left(x_{1}\right) \gamma^{\mu} S_{F}^{e}\left(x_{1}, x_{2}\right) \gamma^{\nu} \psi\left(x_{2}\right) & \\
& \times D_{\mu \xi}\left(x_{1}, x_{3}\right) \Pi_{N}^{\xi \zeta}\left(x_{3}, x_{4}\right) D_{\zeta \nu}\left(x_{4}, x_{2}\right) .
\end{aligned}
$$

Here $\psi$ is the electron wave function, $S_{F}^{e}$ the external-field electron propagator, $D_{\mu \xi}$ the photon propa-

gator and $\Pi_{N}^{\xi \zeta}$ is the nuclear polarization tensor which contains all information of nuclear dynamics. We use units with $\hbar=c=1$ and $e^{2}=4 \pi \alpha$.

In terms of transition charge-current densities, the electron and nuclear parts of Eq. (11) are written as

$$
\bar{\psi}\left(x_{1}\right) \gamma^{\mu} S_{F}^{e}\left(x_{1}, x_{2}\right) \gamma^{\nu} \psi\left(x_{2}\right)=\int \frac{d E}{2 \pi} e^{-i E\left(t_{1}-t_{2}\right)} \sum_{i^{\prime}} \frac{j_{e}^{\mu}\left(\boldsymbol{x}_{1}\right)_{i i^{\prime}} j_{e}^{\nu}\left(\boldsymbol{x}_{2}\right)_{i^{\prime} i}}{E-\omega_{e}+i E_{i^{\prime}}}
$$

and

$$
\Pi_{N}^{\xi \zeta}\left(x_{3}, x_{4}\right)=\int \frac{d \omega}{2 \pi} e^{-i \omega\left(t_{3}-t_{4}\right)} \sum_{I^{\prime}}\left(\frac{J_{N}^{\xi}\left(\boldsymbol{x}_{3}\right)_{I I^{\prime}} J_{N}^{\zeta}\left(\boldsymbol{x}_{4}\right)_{I^{\prime} I}}{\omega-\omega_{N}+i \epsilon}-\frac{J_{N}^{\zeta}\left(\boldsymbol{x}_{4}\right)_{I I^{\prime}} J_{N}^{\xi}\left(\boldsymbol{x}_{3}\right)_{I^{\prime} I}}{\omega+\omega_{N}-i \epsilon}\right)
$$

where $\omega_{e}=E_{i^{\prime}}-E_{i}$ and $\omega_{N}=E_{I^{\prime}}-E_{I}$ are excitation energies of electron and nucleus, respectively. The suffixes $i(I)$ and $i^{\prime}\left(I^{\prime}\right)$ stand for the initial and intermediate states of the electron (nucleus), respectively. 
In the momentum representation, the NP energy shifts due to the ladder and cross diagrams are written as

$$
\begin{aligned}
\Delta E_{N P}^{L}= & -i(4 \pi \alpha)^{2} \int \frac{d \omega}{2 \pi} \int \frac{d \boldsymbol{q}}{(2 \pi)^{3}} \int \frac{d \boldsymbol{q}^{\prime}}{(2 \pi)^{3}} D_{\mu \xi}(\omega, \boldsymbol{q}) D_{\zeta \nu}\left(\omega, \boldsymbol{q}^{\prime}\right) \\
& \times \sum_{i^{\prime}} \frac{j_{e}^{\mu}(-\boldsymbol{q})_{i i^{\prime}} j_{e}^{\nu}\left(\boldsymbol{q}^{\prime}\right)_{i^{\prime} i}}{\omega+\omega_{e}-i E_{i^{\prime}} \epsilon} \sum_{I^{\prime}} \frac{J_{N}^{\xi}(\boldsymbol{q})_{I I^{\prime}} J_{N}^{\zeta}\left(-\boldsymbol{q}^{\prime}\right)_{I^{\prime} I}}{\omega-\omega_{N}+i \epsilon}
\end{aligned}
$$

and

$$
\begin{aligned}
\Delta E_{N P}^{X}= & i(4 \pi \alpha)^{2} \int \frac{d \omega}{2 \pi} \int \frac{d \boldsymbol{q}}{(2 \pi)^{3}} \int \frac{d \boldsymbol{q}^{\prime}}{(2 \pi)^{3}} D_{\mu \xi}(\omega, \boldsymbol{q}) D_{\zeta \nu}\left(\omega, \boldsymbol{q}^{\prime}\right) \\
& \times \sum_{i^{\prime}} \frac{j_{e}^{\mu}(-\boldsymbol{q})_{i i^{\prime}} j_{e}^{\nu}\left(\boldsymbol{q}^{\prime}\right)_{i^{\prime} i}}{\omega+\omega_{e}-i E_{i^{\prime}} \epsilon} \sum_{I^{\prime}} \frac{J_{N}^{\zeta}\left(-\boldsymbol{q}^{\prime}\right)_{I I^{\prime}} J_{N}^{\xi}(\boldsymbol{q})_{I^{\prime} I}}{\omega+\omega_{N}-i \epsilon}
\end{aligned}
$$

respectively. The substitution

$$
\Pi^{\xi \zeta}\left(x_{3}, x_{4}\right) \rightarrow \frac{\rho_{N}\left(\boldsymbol{x}_{3}\right)_{I I}}{m_{p}} \delta^{\xi \zeta} \delta^{4}\left(x_{3}-x_{4}\right)
$$

in Eq. (11) gives the energy correction due to the seagull diagram [13, 14]

$$
\begin{aligned}
\Delta E_{N P}^{S G}= & -i(4 \pi \alpha)^{2} \int \frac{d \omega}{2 \pi} \int \frac{d \boldsymbol{q}}{(2 \pi)^{3}} \int \frac{d \boldsymbol{q}^{\prime}}{(2 \pi)^{3}} D_{\mu \xi}(\omega, \boldsymbol{q}) D_{\zeta \nu}\left(\omega, \boldsymbol{q}^{\prime}\right) \\
& \times \sum_{i^{\prime}} \frac{j_{e}^{\mu}(-\boldsymbol{q})_{i i^{\prime}} j_{e}^{\nu}\left(\boldsymbol{q}^{\prime}\right)_{i^{\prime} i}}{\omega+\omega_{e}-i E_{i^{\prime}} \epsilon} \frac{\rho_{N}\left(\boldsymbol{q}-\boldsymbol{q}^{\prime}\right)_{I I}}{m_{p}} \delta^{\xi \zeta}
\end{aligned}
$$

Here $m_{p}$ is the proton mass, $\delta^{\xi \zeta}$ the Kronecker delta extended to four dimensions with $\delta^{00}=0$, and $\rho_{N}(\boldsymbol{x})_{I I}$ is the ground-state charge distribution of the nucleus. The total NP energy shift is given by the sum, $\Delta E_{N P}^{L}+\Delta E_{N P}^{X}+\Delta E_{N P}^{S G}$.

Substituting the corresponding multipole expansions for the Fourier transforms of the currents, these NP energy shifts are written in terms of the multipole form factors of the electron and nucleus defined by

$$
\begin{aligned}
& <i^{\prime}\left\|m_{\lambda}(q)\right\| i>=\int d \boldsymbol{x} j_{\lambda}(q x)<i^{\prime}\left\|Y_{\lambda}\left(\Omega_{x}\right) \rho_{e}(\boldsymbol{x})\right\| i> \\
& <i^{\prime}\left\|t_{\lambda L}(q)\right\| i>=\int d \boldsymbol{x} j_{L}(q x)<i^{\prime}\left\|\boldsymbol{Y}_{\lambda L}\left(\Omega_{x}\right) \cdot \boldsymbol{j}_{e}(\boldsymbol{x})\right\| i>
\end{aligned}
$$

for the electron, and

$$
\begin{aligned}
& <I^{\prime}\left\|M_{\lambda}(q)\right\| I>=\int d \boldsymbol{x} j_{\lambda}(q x)<I^{\prime}\left\|Y_{\lambda}\left(\Omega_{x}\right) \rho_{N}(\boldsymbol{x})\right\| I> \\
& <I^{\prime}\left\|T_{\lambda L}(q)\right\| I>=\int d \boldsymbol{x} j_{L}(q x)<I^{\prime}\left\|\boldsymbol{Y}_{\lambda L}\left(\Omega_{x}\right) \cdot \boldsymbol{J}_{N}(\boldsymbol{x})\right\| I>
\end{aligned}
$$


for the nucleus. In the above equations, $j_{\lambda}(q x)$ is a spherical Bessel function, $\boldsymbol{Y}_{\lambda L}$ is a vector spherical harmonics and $\lambda$ is the multipolarity of transition. With these substitutions, angular parts of $\boldsymbol{q}$ and $\boldsymbol{q}^{\prime}$ as well as $\omega$ integrations in Eqs. (4), (5) and (7) can be carried out analytically. Integrations with respect to $q$ and $q^{\prime}$ are carried out numerically. Nuclear-polarization energy shifts are thus given by the sum of these double integrals over the nuclear and electron intermediate states. For the seagull contribution, the summation is only over the electron intermediate states.

In the following, we shall give formula for the NP energy both in the Feynman and Coulomb gauges. We restrict ourselves to $I^{\pi}=0^{+}$for the spin-parity of the nuclear ground state. The spin-parity of the nuclear intermediate state $I^{\prime \pi}$ is in this case equal to the spin-parity of the transition $\lambda^{\pi}$.

\section{A. The Feynman gauge}

The photon propagator in the Feynman gauge is given by

$$
D_{\mu \xi}^{F}(\omega, \boldsymbol{q})=-\frac{g_{\mu \xi}}{q^{2}+i \epsilon}
$$

Here the metric tensor $g_{\mu \xi}$ is defined by $g_{00}=1$ and $g_{i i}=-1$. With this propagator, the NP energies due to the ladder, cross and seagull terms are given by

$$
\begin{aligned}
& \Delta E_{N P}^{L}=-\sum_{i^{\prime} I^{\prime}} \frac{(4 \pi \alpha)^{2}}{(2 i+1)\left(2 I^{\prime}+1\right)}\left(\frac{2}{\pi}\right)^{2} \mathcal{P} \int_{0}^{\infty} d q \int_{0}^{\infty} d q^{\prime} I_{+}\left(q, q^{\prime}\right) \mathcal{W}_{F}^{L}(q) \mathcal{W}_{F}^{L}\left(q^{\prime}\right) \delta_{I^{\prime} \lambda}, \\
& \Delta E_{N P}^{X}=-\sum_{i^{\prime} I^{\prime}} \frac{(4 \pi \alpha)^{2}}{(2 i+1)\left(2 I^{\prime}+1\right)}\left(\frac{2}{\pi}\right)^{2} \mathcal{P} \int_{0}^{\infty} d q \int_{0}^{\infty} d q^{\prime} I_{-}\left(q, q^{\prime}\right) \mathcal{W}_{F}^{X}(q) \mathcal{W}_{F}^{X}\left(q^{\prime}\right) \delta_{I^{\prime} \lambda}, \\
& \Delta E_{N P}^{S G}=-\sum_{i^{\prime}} \frac{(4 \pi \alpha)^{2}}{(2 i+1)}\left(\frac{2}{\pi}\right)^{2} \mathcal{P} \int_{0}^{\infty} d q \int_{0}^{\infty} d q^{\prime} I_{S G}\left(q, q^{\prime}\right) \mathcal{W}_{S G}^{F}\left(q, q^{\prime}\right),
\end{aligned}
$$

where $\mathcal{P}$ denotes the Cauchy principal value of any improper integral. The $q$ and $q^{\prime}$ integrals may be improper in case of the NP calculation for the electron excited states.

In the above expressions, $I_{+}, I_{-}$and $I_{S G}$ are functions written as

$$
\begin{aligned}
I_{ \pm}\left(q, q^{\prime}\right)= & \frac{q q^{\prime}}{2\left(\tilde{\omega}_{e}+q\right)\left(\tilde{\omega}_{e}+q^{\prime}\right)\left(\omega_{N}+q\right)\left(\omega_{N}+q^{\prime}\right)} \\
& \times\left\{\operatorname{sgn}\left(E_{i^{\prime}}\right)\left[\frac{\tilde{\omega}_{e} \omega_{N}}{q+q^{\prime}}+\left(\tilde{\omega}_{e}+\omega_{N}+q+q^{\prime}\right)\right] \pm \frac{q q^{\prime}}{q+q^{\prime}} \pm \theta\left( \pm E_{i^{\prime}}\right) \frac{2 q q^{\prime}}{\omega_{N}+\tilde{\omega}_{e}}\right\}, \\
I_{S G}\left(q, q^{\prime}\right)= & \operatorname{sgn}\left(E_{i^{\prime}}\right) \frac{1}{2 m_{p}} \frac{q q^{\prime}\left(\tilde{\omega}_{e}+q+q^{\prime}\right)}{\left(q+q^{\prime}\right)\left(q+\tilde{\omega}_{e}\right)\left(q^{\prime}+\tilde{\omega}_{e}\right)},
\end{aligned}
$$

where $\tilde{\omega}_{e}=\operatorname{sgn}\left(E_{i^{\prime}}\right) \omega_{e}$, while $\mathcal{W}_{L}^{F}(q), \mathcal{W}_{X}^{F}(q)$ and $\mathcal{W}_{S G}^{F}(q)$ are written in terms of the electron and nuclear form factors as 


$$
\begin{aligned}
\mathcal{W}_{L}^{F}(q) & =\sum_{\lambda}\left[<i^{\prime}\left\|m_{\lambda}(q)\right\| i><I^{\prime}\left\|M_{\lambda}(q)\right\| I>\right. \\
& \left.-\sum_{L=\lambda-1}^{\lambda+1}(-1)^{L+1-\lambda}<i^{\prime}\left\|t_{\lambda L}(q)\right\| i><I^{\prime}\left\|T_{\lambda L}(q)\right\| I>\right], \\
\mathcal{W}_{X}^{F}(q) & =\sum_{\lambda}\left[<i^{\prime}\left\|m_{\lambda}(q)\right\| i><I^{\prime}\left\|M_{\lambda}(q)\right\| I>\right. \\
& \left.+\sum_{L=\lambda-1}^{\lambda+1}<i^{\prime}\left\|t_{\lambda L}(q)\right\| i><I^{\prime}\left\|T_{\lambda L}(q)\right\| I>\right], \\
\mathcal{W}_{S G}^{F}\left(q, q^{\prime}\right) & =\sum_{\lambda} \sum_{L=\lambda-1}^{\lambda+1}<i^{\prime}\left\|t_{\lambda L}(q)\right\| i><I\left\|M_{L}\left(q, q^{\prime}\right)\right\| I><i^{\prime}\left\|t_{\lambda L}\left(q^{\prime}\right)\right\| i>.
\end{aligned}
$$

The seagull term contains the Fourier transform of the nuclear ground state,

$$
<I\left\|M_{L}\left(q, q^{\prime}\right)\right\| I>=\int r^{2} d r \rho_{N}(r)_{I I} j_{L}(q r) j_{L}\left(q^{\prime} r\right) .
$$

\section{B. The Coulomb gauge}

The photon propagator in the Coulomb gauge is given by

$$
D_{00}^{C}(\omega, \boldsymbol{q})=\frac{1}{q^{2}+i \epsilon}, \quad D_{i j}^{C}(\omega, \boldsymbol{q})=\frac{1}{q^{2}+i \epsilon}\left(\delta_{i j}-\frac{q_{i} q_{j}}{|\boldsymbol{q}|^{2}}\right)
$$

and $\left(\delta_{i j}-\frac{q_{i} q_{j}}{|\boldsymbol{q}|^{2}}\right)$ in $D_{i j}^{C}$ projects out both transverse parts of electronic and nuclear currents.

Making use of the relation

$$
\boldsymbol{j}_{e}^{T}(-\boldsymbol{q}) \cdot \boldsymbol{J}_{N}^{T}(\boldsymbol{q})=\boldsymbol{j}_{e}(-\boldsymbol{q}) \cdot \boldsymbol{J}_{N}(\boldsymbol{q})+\frac{\omega_{e} \omega_{N}}{q^{2}} \rho_{e}(-\boldsymbol{q}) \rho_{N}(\boldsymbol{q}),
$$

we obtain the NP energies for the ladder, cross and seagull terms:

$$
\begin{aligned}
\Delta E_{N P}^{L} & =-\sum_{i^{\prime} I^{\prime}} \frac{(4 \pi \alpha)^{2}}{(2 i+1)\left(2 I^{\prime}+1\right)} \mathcal{P} \int_{0}^{\infty} d q \int_{0}^{\infty} d q^{\prime}\left\{\frac{\theta\left(E_{i^{\prime}}\right)}{\tilde{\omega}_{e}+\omega_{N}} \mathcal{W}_{L}^{C}(q) \mathcal{W}_{L}^{C}\left(q^{\prime}\right)\right. \\
& \left.+I_{+}\left(q, q^{\prime}\right) \mathcal{W}_{T}^{C}(q) \mathcal{W}_{T}^{C}\left(q^{\prime}\right)+I_{+}^{L T}\left(q^{\prime}\right) \mathcal{W}_{L}^{C}(q) \mathcal{W}_{T}^{C}\left(q^{\prime}\right)\right\} \delta_{I^{\prime} \lambda}, \\
\Delta E_{N P}^{X} & =-\sum_{i^{\prime} I^{\prime}} \frac{(4 \pi \alpha)^{2}}{(2 i+1)\left(2 I^{\prime}+1\right)} \mathcal{P} \int_{0}^{\infty} d q \int_{0}^{\infty} d q^{\prime}\left\{\frac{-\theta\left(-E_{i^{\prime}}\right)}{\tilde{\omega}_{e}+\omega_{N}} \mathcal{W}_{L}^{C}(q) \mathcal{W}_{L}^{C}\left(q^{\prime}\right)\right. \\
& \left.+I_{-}\left(q, q^{\prime}\right) \mathcal{W}_{T}^{C}(q) \mathcal{W}_{T}^{C}\left(q^{\prime}\right)+I_{-}^{L T}\left(q^{\prime}\right) \mathcal{W}_{L}^{C}(q) \mathcal{W}_{T}^{C}\left(q^{\prime}\right)\right\} \delta_{I^{\prime} \lambda}, \\
\Delta E_{N P}^{S G} & =-\sum_{i^{\prime}} \frac{(4 \pi \alpha)^{2}}{(2 i+1)}\left(\frac{2}{\pi}\right)^{2} \mathcal{P} \int_{0}^{\infty} d q \int_{0}^{\infty} d q^{\prime} I_{S G}\left(q, q^{\prime}\right) \mathcal{W}_{S G}^{C}\left(q, q^{\prime}\right) .
\end{aligned}
$$


In the above expressions, $I_{+}^{L T}\left(q^{\prime}\right)$ and $I_{-}^{L T}\left(q^{\prime}\right)$ come from the interference between the longitudinal and transverse contributions and given by

$$
I_{ \pm}^{L T}\left(q^{\prime}\right)= \pm \frac{\operatorname{sgn}\left(E_{i^{\prime}}\right) q^{\prime}\left(\tilde{\omega}_{e}+\omega_{N}\right) \pm \theta\left( \pm E_{i^{\prime}}\right) 2 q^{\prime 2}}{\left(q^{\prime}+\tilde{\omega}_{e}\right)\left(q^{\prime}+\omega_{N}\right)\left(\tilde{\omega}_{e}+\omega_{N}\right)} .
$$

In the Coulomb gauge, $\mathcal{W}_{L}^{C}(q), \mathcal{W}_{L}^{C}(q)$ and $\mathcal{W}_{L}^{C}(q)$ are given by

$$
\begin{aligned}
\mathcal{W}_{L}^{C}(q) & =\sum_{\lambda}<i^{\prime}\left\|m_{\lambda}(q)\right\| i><I^{\prime}\left\|M_{\lambda}(q)\right\| I> \\
\mathcal{W}_{T}^{C}(q) & =-\sum_{\lambda}\left[\frac{\omega_{e} \omega_{N}}{q^{2}} \mathcal{W}_{L}^{C}(q)+\sum_{L=\lambda-1}^{\lambda+1}<i^{\prime}\left\|t_{\lambda L}(q)\right\| i><I^{\prime}\left\|T_{\lambda L}(q)\right\| I>\right] \\
\mathcal{W}_{S G}^{C}\left(q, q^{\prime}\right) & =\sum_{\lambda}\left[\sum_{L=\lambda \pm 1}\left(<i^{\prime}\left\|u_{\lambda L}(q)\right\| i><I\left\|M_{L}\left(q, q^{\prime}\right)\right\| I><i^{\prime}\left\|u_{\lambda L}\left(q^{\prime}\right)\right\| i>\right)\right. \\
& \left.+<i^{\prime}\left\|t_{\lambda \lambda}(q)\right\| i><I\left\|M_{\lambda}\left(q, q^{\prime}\right)\right\| I><i^{\prime}\left\|t_{\lambda \lambda}\left(q^{\prime}\right)\right\| i>\right]
\end{aligned}
$$

with

$$
\begin{aligned}
& <i^{\prime}\left\|u_{\lambda \lambda-1}(q)\right\| i>=<i^{\prime}\left\|t_{\lambda-1}(q)\right\| i>-i \sqrt{\frac{\lambda}{2 \lambda+1}} \frac{\omega_{e}}{q}<i^{\prime}\left\|m_{\lambda}(q)\right\| i> \\
& <i^{\prime}\left\|u_{\lambda \lambda+1}(q)\right\| i>=<i^{\prime}\left\|t_{\lambda+1}(q)\right\| i>-i \sqrt{\frac{\lambda+1}{2 \lambda+1}} \frac{\omega_{e}}{q}<i^{\prime}\left\|m_{\lambda}(q)\right\| i>
\end{aligned}
$$

\section{Electron wave functions}

The radial Dirac equations for the electron are written as

$$
\begin{aligned}
& \left(\frac{d}{d r}+\frac{\kappa}{r}\right) G_{E, \kappa}=\left[m_{e}+E-V(r)\right] F_{E, \kappa}(r), \\
& \left(\frac{d}{d r}-\frac{\kappa}{r}\right) F_{E, \kappa}=\left[m_{e}-E+V(r)\right] G_{E, \kappa}(r),
\end{aligned}
$$

where the potential $V(r)$ is obtained from the the ground-state charge distribution of ${ }^{208} \mathrm{~Pb}$, which is assumed to be a two parameter Fermi distribution

$$
\rho_{N}(r)_{I I}=\frac{\rho_{0}}{1+\exp \left[\left(r-R_{0}\right) / a\right]}
$$

with $R_{0}=6.6477 \mathrm{fm}$ and $a=0.5234 \mathrm{fm}$ 10. These equations are solved numerically by using the fourth-order Runge-Kutta method. For both positive and negative energy continuum states, the radial functions are normalized by the conditions 


$$
\begin{aligned}
& G_{E, \kappa} \underset{r \rightarrow \infty}{\longrightarrow}\left(\frac{\left|E+m_{e}\right|}{\pi p}\right)^{1 / 2} \sin (p r+\delta), \\
& F_{E, \kappa} \underset{r \rightarrow \infty}{\longrightarrow}\left(\frac{\left|E-m_{e}\right|}{\pi p}\right)^{1 / 2} \cos (p r+\delta),
\end{aligned}
$$

with $p=\sqrt{E^{2}-m_{e}^{2}}$. The bound-state wave functions are normalized as $\left[\int_{0}^{\infty}\left(G_{n \kappa}^{2}+F_{n \kappa}^{2}\right) d r=1\right]$.

Transition form factors for the electron, (8) and (9), are calculated using the formula given in Ref. [10]. They are stored in the computer with six different step sizes of $\Delta q$ depending on the electron energy $E_{i^{\prime}}$. In Fig. 2 we show the $E 1$ charge form factors $<E_{i^{\prime}}, p_{1 / 2}\left\|m_{1}(q)\right\| 1 s_{1 / 2}>$ of the electron with three different energies, $E_{i^{\prime}}=2,6$ and $10 \mathrm{MeV}$. One finds that they have sharp peaks at $q=E_{i^{\prime}}$ and decrease rapidly as $q$ increases. Numerical integrations in Eqs. (13)-(15) and (24)-(26) are performed by Simpson's one-third rule.

Most of the NP correction comes from the continuum states with energies greater than $m_{e}$ and energies less than $-m_{e}$. Summation over the electron states $i^{\prime}$ in Eqs. (13)-(15) and Eqs. (24)-(26) implies an integration with respect to $E_{i^{\prime}}$. The $E_{i^{\prime}}$ integration is carried out by using the Gauss-Legendre quadrature over the intervals $-250 \mathrm{MeV}<E_{i^{\prime}}<-m_{e}$ and $m_{e}<E_{i^{\prime}}<250 \mathrm{MeV}$. Accuracy of the numerical results is checked by comparing them with the results of Simpson's rule. We also included the electron bound states for $n^{\prime} \leq 7$ in the calculation.

\section{RPA calculation of nuclear charge and current densities}

The random-phase approximation is used to describe the nuclear excitations. The calculation we employed is the same as those performed earlier in Ref. [10], i.e., the same single-particle basis, the same particle-hole configuration of approximately a full $3 \hbar \omega$ space and the same Migdal force [15] parameters to describe nuclear two-body interaction. Nuclear transition form factors, (10) and (11), are calculated by using the formula given in Ref. [10].

The calculated charge and magnetic current densities are examined by comparing with experimental $B(E \lambda)$ and $B(M \lambda)$ using the following relations;

$$
B\left(E \lambda: I \rightarrow I^{\prime}\right)=\frac{1}{2 I+1}\left|e \int \rho_{N \lambda}^{I^{\prime} I}(r) r^{\lambda+2} d r\right|^{2}
$$

and

$$
B\left(M \lambda: I \rightarrow I^{\prime}\right)=\frac{1}{2 I+1} \frac{\lambda}{\lambda+1}\left|e \int J_{N \lambda \lambda}^{I^{\prime} I}(r) r^{\lambda+2} d r\right|^{2}
$$


In Table I, we compared the energy-weighted sum of $B(E \lambda)$ over the RPA states with the classical energy-weighted sum-rule value (EWSR) of Ref. [16. The results for the $E 0$ and $E 1$ transitions exceed the EWSR by $20 \%$ and $10 \%$, respectively while the results for the $E 2$ and $E 3$ transitions agree well with the EWSR. For the E4 and E5 transitions, our results exhaust only $50 \%$ of the EWSR. This may be due to the insufficient configuration space for the $E 4$ and $E 5$ calculations.

On the other hand, there is no experimental constraint imposed on the nuclear electric current. However, the electromagnetic current should satisfy the continuity equation required by charge conservation;

$$
\frac{\partial}{\partial t} \hat{\rho}_{N}+\nabla \hat{\boldsymbol{J}}_{N}=0
$$

Using the nuclear Hamiltonian $H_{N}$, we rewrite it as

$$
i\left[H_{N}, \hat{\rho}_{N}\right]+\nabla \hat{\boldsymbol{J}}_{N}=0
$$

Taking the matrix element of Eq. (41) between the initial and the final nuclear states, we obtain charge conservation condition;

$$
i \omega_{N} \rho_{N \lambda}^{I^{\prime} I}(r)=-\sqrt{\frac{\lambda}{2 \lambda+1}}\left(\frac{d}{d r}-\frac{\lambda-1}{r}\right) J_{N \lambda \lambda-1}^{I^{\prime} I}(r)+\sqrt{\frac{\lambda+1}{2 \lambda+1}}\left(\frac{d}{d r}+\frac{\lambda+2}{r}\right) J_{N \lambda \lambda+1}^{I^{\prime} I}(r) .
$$

Here, $\omega_{N}=E_{I^{\prime}}-E_{I}$ is the energy of nuclear excitation.

For any kind of model calculation involving the nuclear current, it is necessary for the model to satisfy the charge conservation condition in order to observe the gauge invariance [13]. Unfortunately, the charge-current densities constructed from the present RPA calculation do not satisfy the charge conservation of Eq. (42). The violation of the charge conservation comes from the inconsistency of using empirical single-particle energies together with the impulse charge-current operators, as is discussed in Refs. 10,17. It is desirable if one could construct a microscopic self-consistent model together with the nuclear current satisfying the charge conservation which is realistic enough to reproduce the observed spectra and $B(E \lambda)$ values. However, the refinement of calculation will be left for a future work and at present we are satisfied with the fact that the calculated NP energies show only a small gauge violation even though the empirical single-particle energies are used in the RPA calculation.

\section{RESULTS AND DISCUSSION}

NP energy shifts are obtained by computing an energy shift for each of the RPA excitations and summing the results. Our calculation gives 38, 129, 160, 222, 202, 218 and 70 nuclear states for the $0^{+}$, 
$1^{-}, 2^{+}, 3^{-}, 4^{+}, 5^{-}$and $1^{+}$excitations, respectively. Figure 3 shows the NP energy spectra of the $1 s_{1 / 2}$ state for the respective nuclear spin-parities. These spectra are calculated in the Coulomb gauge with the Coulomb and transverse parts of the electromagnetic interaction. They are very similar to the RPA spectra of $B(E \lambda)$ and $B(M 1)$.

Table II summarizes the NP energies of the $1 s_{1 / 2}$ state for ${ }_{82}^{208} \mathrm{~Pb}^{81+}$. The 1 st column denotes nuclear spin-parities. The entries in the 2nd column indicate the contributions to the NP energy from the ladder, cross and seagull terms as well as those of the positive and negative energy intermediate-states of the electron. The 3rd column shows the NP energies in the Feynman gauge, while the 4th column shows the NP energies in the Coulomb gauge. The transverse contributions are included in both columns. The 5th column shows the Coulomb NP energies without the transverse contribution (hereafter referred to as CNP). The 6th column shows the results of the previous NP calculation in the Feynman gauge assuming a collective model for the nuclear excitations [12]. The 7th column is the CNP of the same calculation. Finally, the last column shows the CNP calculated by another group [8].

In Table II, it should be noted that the positive and negative energy continuum states of the electron always contribute to a NP energy with opposite sign, the fact that is already observed in Refs. [6, 12]. It is also understood from the fact that the contribution of the negative energy electron describes the blocking of the response of vacuum due to the occupied state of the atomic electron. In electronic atoms the virtual pair creation requires energy of only $2 m_{e}$, which is smaller than the typical excitation energy of a nucleus and is correspondingly important.

The 5th column (the present CNP) may be compared with the 7th and 8th columns of the previous calculations. All three calculations show very good agreement with one another. The agreement indicates that provided the $B(E \lambda)$ values are similarly chosen, CNP of electronic atoms is not very sensitive to the detail of transition charge densities. The monopole NP energy shows some difference between the calculations. It is $-4.0 \mathrm{meV}$ ( 5 th column), $-7.2 \mathrm{meV}$ (7th column) and $-3.3 \mathrm{meV}$ (8th column), respectively. The difference is mainly caused by the Dirac-electron wave functions used; those for the finite charge distribution are used in the 5th and 8th columns, while those for a point charge are used in the 7th column. The difference is conspicuous only for the monopole NP energy because the nuclear monopole transition-potential exists only inside the nucleus where the electron-wave functions generated from the point charge and the finite charge distribution differ appreciably.

The dipole NP energy also shows some difference, i.e., it is $-20.3 \mathrm{meV}$ (the 4 th column), $-19.5 \mathrm{meV}$ (the 6 th column) and $-17.6 \mathrm{meV}$ (the 7 th column), respectively. The difference between the 4 th and the 
7th columns arises from the fact that the energy-weighted sum of $B(E 1)$ over the RPA states exceeds the classical EWSR value by $10 \%$ (see Table I).

The NP energies including the transverse effect (the 3rd column) can be compared with the results of Ref. [12] (the 6th column), both of which were calculated in the Feynman gauge. Agreement between the two calculations is good except for the nuclear monopole excitation. Here again, we may conclude that NP energies of heavy electronic atoms are not very sensitive to the detail of transition current densities.

An important feature of the present calculation, which is in fact crucial for the numerical estimate of NP energies, is that there exists a large violation of gauge invariance in the NP energy-shifts as far as only the ladder and cross diagrams of Figs. 1(a) and 1(b) are taken into account. By using the minimal prescription, the nonrelativistic electromagnetic interaction involves the square of vector potential called a seagull term coming from the kinetic energy, and this term is necessary for the gauge invariance in a nonrelativistic system [13,14. It is interesting to investigate whether the inclusion of the seagull term restores the gauge invariance of the present RPA calculation. (A proof of the gauge invariance is given in the Appendix.)

As is seen from Table II, this is nicely confirmed numerically. With the ladder and cross diagrams, the NP energy for the $1 s_{1 / 2}$ was $+1.5 \mathrm{meV}$ in the Feynman gauge, while $-32.7 \mathrm{meV}$ in the Coulomb gauge. The gauge dependence was $34.2 \mathrm{meV}$. However, after the inclusion of the seagull term, it is $-38.2 \mathrm{meV}$ in the Feynman gauge and $-37.0 \mathrm{meV}$ in the Coulomb gauge. The gauge dependence is reduced to 1.2 meV. This small gauge dependence shows that the seagull term is quite important in restoring the gauge invariance of the NP calculation. The fact also implies that the use of empirical single-particle energies in the RPA calculation does not introduce a serious violation of gauge invariance into the NP energies.

In Table II, we recognize that the nuclear dipole states give predominant NP contributions. The transverse contribution in particular is very large if the contribution from the positive and negative energy states are separately considered. For example, in the Coulomb gauge, the Coulomb plus transverse contribution from the ladder and cross diagrams is $-120.5 \mathrm{meV}$, while the corresponding contribution without the transverse term is $-37.0 \mathrm{meV}$. The reason for this fact is understood as follows. For simplicity, we disregard the retardation of the interaction. Then Eq. (22) gives the Coulomb-Breit potentials for electron:

$$
\begin{aligned}
& V_{C}(\boldsymbol{r})_{I^{\prime} I}=\int d \boldsymbol{r}^{\prime} \frac{\rho_{N}\left(\boldsymbol{r}^{\prime}\right)_{I^{\prime} I}}{\left|\boldsymbol{r}-\boldsymbol{r}^{\prime}\right|} \\
& V_{T}(\boldsymbol{r})_{I^{\prime} I}=\int d \boldsymbol{r}^{\prime} \frac{\boldsymbol{J}_{N}^{T}\left(\boldsymbol{r}^{\prime}\right)_{I^{\prime} I}}{\left|\boldsymbol{r}-\boldsymbol{r}^{\prime}\right|} .
\end{aligned}
$$


In Fig. 4 the radial parts of the potentials due to the $1^{-}, 14.6 \mathrm{MeV}$ state are plotted after multipole expansions of Eqs. (43) and (44). The Coulomb potential is larger than both of the transverse potentials in the region $\mathrm{r}<60 \mathrm{fm}$, while the transverse potential with $\lambda=1, L=0$ becomes larger than the Coulomb potential in the region $r>60 \mathrm{fm}$. This long-range nature of the transverse potential explains the fact that the $E 1$ transverse contribution is dominant for electronic atoms because the Bohr radius of the $1 s_{1 / 2}$ state is much larger than $60 \mathrm{fm}$. Since the radial dependence of the Coulomb potential (transverse potential) with multipolarity $\lambda$ is $1 / r^{\lambda+1}\left(1 / r^{\lambda}\right)$ outside the nuclear region, the magnitude of the potentials in the region of the Bohr radius decreases as the multipolarity increases. Therefore, for multipoles other than the dipole, i.e., $\lambda^{\pi}=2^{+}, 3^{-}, 4^{+}$and $5^{-}$, the NP energy is mainly determined by the overlap between the electron transition density and nuclear multipole potential in the region of the nuclear surface, hence the transverse contribution is small compared with the Coulomb contribution. The fact also explains that the Coulomb contribution is predominant for heavy muonic atoms in any multipolarities because the overlap near the nucleus is always significant due to the small muon Bohr-radius [10].

The dominance of the E1 contribution in the NP energy can be seen more clearly in the spectral density of the NP contribution from a particular nuclear excitation as a function of electron energy. In Fig. 5, these spectral functions in the Coulomb gauge are shown for three different nuclear states; (a) $0^{+}(13.3 \mathrm{MeV})$, (b) $1^{-}(14.6 \mathrm{MeV})$ and (c) $2^{+}(10.2 \mathrm{MeV})$. In each panel, the solid line shows the spectral function including the transverse contribution, while the dotted line shows the result without the transverse effect. The NP energy due to each of the nuclear states in Fig. 3 is given by the integral of the corresponding spectral function over the electron energy. One can see that the low energy region of the spectral function for the $1^{-}$state (Fig. $\left.5(\mathrm{~b})\right)$ is different from the other two. The E1 spectrum shows a peak at threshold $\left(E_{i^{\prime}}=m_{e}\right)$. It should be reminded that only the $<I^{\prime}\left\|T_{10}(q)\right\| I>$ and $<i^{\prime}\left\|t_{10}(q)\right\| i>$ are nonvanishing at $q=0$, so that the large overlap between these transverse form factors is guaranteed in the low momentum region.

For the excited $L$-shell electrons, we can repeat the discussion that the transverse $E 1$ multipole plays a crucial role in the NP effects of hydrogenlike atoms and essentially determines the magnitude of the NP effects. The total NP shifts for the $1 s_{1 / 2}, 2 s_{1 / 2}, 2 p_{1 / 2}$, and $2 p_{3 / 2}$ states are summarized in Table III and are compared with the Coulomb NP energies. The relative importance of the transverse contribution is about $10-20 \%$ effect for the $1 s_{1 / 2}$ and $2 s_{1 / 2}$ states, and the level shifts for the $2 p_{1 / 2}$ and $2 p_{3 / 2}$ states are negligible.

Since the nuclear dipole states have predominant NP contributions, we must note here effects of the 
spurious center-of-mass motion of the nucleus on the NP energies. The present Migdal force brings down the lowest $1^{-}$state to the imaginary eigenvalue of $1.32 i \mathrm{MeV}$. Since this $1^{-}$state carries most of the spurious center-of-mass motion, we excluded this nuclear state from the NP calculation. The $0.7 \%$ of the spurious center-of-mass motion remains in the rest of the $1^{-}$states, whose effects on the NP energies are negligible. Thus our results for NP energy due to the cross and ladder diagrams contain intrinsic excitations only. On the other hand, the seagull contributions calculated by using Eq. (21) contain both of the effects of intrinsic excitations and center-of-mass motion. The seagull contribution coming from the center-of-mass motion must be eliminated for the dipole mode. This was achieved by using the effective dipole charges $e_{p}=N / A, e_{n}=-Z / A$ instead of using the true charges $e_{p}=1, e_{n}=0$.

\section{SUMMARY}

We have calculated the NP energy shifts for the hydrogenlike ${ }_{82}^{208} \mathrm{~Pb}^{81+}$ taking into account the effects of the electron in the negative energy continuum besides the usual contributions of the electron excited into higher unoccupied orbitals. The evaluation of the NP energies contains the seagull graph as well as the ladder and cross diagrams. The Dirac-electron wave functions were solved in the Coulomb potential with a finite charge distribution for the nuclear ground state and the RPA wave functions were employed for the nuclear excited states.

The results presented in the previous section can be summarized as follows. 1)In the Feynman (Coulomb) gauge, we obtained the NP energies of $-38.2(-37.0),-6.7(-6.4),-0.2(-0.2)$ and $+0.0(+0.0)$ meV for the $1 s_{1 / 2}, 2 s_{1 / 2}, 2 p_{1 / 2}$ and $2 p_{3 / 2}$ states, respectively. 2) The nuclear dipole states have predominant contributions to the NP energy of heavy electronic atoms. In particular the exchange of transverse photon plays a crucial role. The net E1 contribution, however, becomes quite small due to the cancellation between the contributions of the electron in the positive and negative energy intermediate states. 3) Due to the cancellation, the transverse contribution to the total NP energy shift is about $10 \%$ of the Coulomb contribution for the $1 s_{1 / 2}$ state. 4) The NP shifts of electronic atoms have serious gauge dependence if one calculates them with only the ladder and cross diagrams of the two-photon exchange processes. Indeed the NP energies for the $1 s_{1 / 2}$ state due to these diagrams are $+1.5 \mathrm{meV}$ and -32.7 meV in the Feynman and Coulomb gauges, respectively. The inclusion of the seagull graph gives the NP energies of $-38.2 \mathrm{meV}$ and $-37.0 \mathrm{meV}$. The seagull graph is quite important in restoring the gauge invariance of the NP calculation. 5) The NP energy for electronic atoms is not sensitive to the details of the 
transition charge and current densities because it is primarily given by the overlap between an electron transition density and a nuclear multipole potential in the region outside the nucleus. 6) The present RPA calculation using the empirical single-particle energies together with the impulse charge-current does not seem to introduce serious gauge dependence into the NP calculation.

\section{ACKNOWLEDGMENTS}

We would like to thank N. Yamanaka and A. Ichimura for arousing our interest in the electronic-atom NP calculation. 


\section{APPENDIX A: GAUGE INVARIANCE}

To prove the gauge invariance of the NP energy, we write the sum of the ladder and cross contributions as follows,

$$
\begin{aligned}
\Delta E_{N P}^{L}+\Delta E_{N P}^{X}= & -i \frac{(4 \pi \alpha)^{2}}{2} \int \frac{d \omega}{2 \pi} \int \frac{d \boldsymbol{q}}{(2 \pi)^{3}} \int \frac{d \boldsymbol{q}^{\prime}}{(2 \pi)^{3}} \\
& \Pi_{e}^{\mu \nu}\left(\omega, \boldsymbol{q}, \boldsymbol{q}^{\prime}\right) D_{\mu \xi}(\omega, q) D_{\zeta \nu}\left(\omega, q^{\prime}\right) \Pi_{N}^{\xi \zeta}\left(\omega, \boldsymbol{q}, \boldsymbol{q}^{\prime}\right),
\end{aligned}
$$

where $\Pi_{e}^{\mu \nu}\left(\omega, \boldsymbol{q}, \boldsymbol{q}^{\prime}\right)$ and $\Pi_{N}^{\xi \zeta}\left(\omega, \boldsymbol{q}, \boldsymbol{q}^{\prime}\right)$ are the electronic and nuclear polarization tensors defined by

$$
\begin{aligned}
& \Pi_{e}^{\mu \nu}\left(\omega, \boldsymbol{q}, \boldsymbol{q}^{\prime}\right)=\sum_{i^{\prime}}\left(\frac{j_{e}^{\mu}(-\boldsymbol{q})_{i i^{\prime}} j_{e}^{\nu}\left(\boldsymbol{q}^{\prime}\right)_{i^{\prime} i}}{\omega+\omega_{e}-i E_{i^{\prime}} \epsilon}-\frac{j_{e}^{\nu}\left(\boldsymbol{q}^{\prime}\right)_{i i^{\prime}} j_{e}^{\mu}(-\boldsymbol{q})_{i^{\prime} i}}{\omega-\omega_{e}+i E_{i^{\prime}} \epsilon}\right), \\
& \Pi_{N}^{\xi \zeta}\left(\omega, \boldsymbol{q}, \boldsymbol{q}^{\prime}\right)=\sum_{I^{\prime}}\left(\frac{J_{N}^{\xi}(\boldsymbol{q})_{I I^{\prime}} J_{N}^{\zeta}\left(-\boldsymbol{q}^{\prime}\right)_{I^{\prime} I}}{\omega-\omega_{N}+i \epsilon}-\frac{J_{N}^{\zeta}\left(-\boldsymbol{q}^{\prime}\right)_{I I^{\prime}} J_{N}^{\xi}(\boldsymbol{q})_{I^{\prime} I}}{\omega+\omega_{N}-i \epsilon}\right) .
\end{aligned}
$$

Photon propagators in the Feynman and Coulomb gauges are related to each other by

$$
D_{\mu \xi}^{C}(q, \omega)=D_{\mu \xi}^{F}(q, \omega)-\frac{1}{q^{2}+i \epsilon}\left(\frac{q_{\mu} q_{\xi}-\omega\left(q_{\mu} g_{\xi 0}+q_{\xi} g_{\mu 0}\right)}{q^{2}}\right) .
$$

If both $q_{\mu} \Pi_{e}^{\mu \nu}=0$ and $q_{\xi} \Pi_{N}^{\xi \zeta}=0$ are satisfied, it is easy to see that the Feynman and Coulomb gauges give the same result for the NP contributions given by Eq. (A1).

Multiplying both sides of the electron polarization tensor by $q_{\mu}$, and using the continuity equation of the charge conservation, one obtains

$$
\begin{aligned}
q_{\mu} \Pi_{e}^{\mu \nu}\left(\omega, \boldsymbol{q}, \boldsymbol{q}^{\prime}\right) & =\sum_{i^{\prime}}\left(<i\left|\hat{\rho}_{e}(-\boldsymbol{q})\right| i^{\prime}><i^{\prime}\left|\hat{j}_{e}^{\nu}\left(\boldsymbol{q}^{\prime}\right)\right| i>-<i\left|\hat{j}_{e}^{\nu}\left(\boldsymbol{q}^{\prime}\right)\right| i^{\prime}><i^{\prime}\left|\hat{\rho}_{e}(-\boldsymbol{q})\right| i>\right) \\
& =<i\left|\left[\hat{\rho}_{e}(-\boldsymbol{q}), \hat{j}_{e}^{\nu}\left(\boldsymbol{q}^{\prime}\right)\right]\right| i>
\end{aligned}
$$

In deriving the second equality, we have assumed the completeness of the intermediate states of the electron. For the electromagnetic charge and current operators

$$
\hat{\rho}_{e}(-\boldsymbol{q})=\left(\begin{array}{ll}
1 & 0 \\
0 & 1
\end{array}\right) e^{i \boldsymbol{q} \cdot \boldsymbol{r}}, \quad \hat{j}_{e}\left(\boldsymbol{q}^{\prime}\right)=\left(\begin{array}{cc}
0 & \boldsymbol{\sigma} \\
\boldsymbol{\sigma} & 0
\end{array}\right) e^{-i \boldsymbol{q}^{\prime} \cdot \boldsymbol{r}}
$$

used with the Dirac-electron wave functions, the commutation relation in Eq. (A5) vanishes. Hence the gauge invariance $q_{\mu} \Pi_{e}^{\mu \nu}\left(\omega, \boldsymbol{q}, \boldsymbol{q}^{\prime}\right)=0$ for the electronic polarization tensor follows.

For the nuclear polarization tensor, we can obtain the similar form to Eq. (A5) by assuming the charge conservation as well as the completeness relation. In the present calculation, the impulse charge and current operators

$$
\hat{\rho}_{N}(-\boldsymbol{q})=\sum_{i}^{Z} e^{i \boldsymbol{q} \cdot \boldsymbol{r}_{i}}, \quad \hat{J}_{N}(\boldsymbol{q})=\sum_{i}^{Z} \frac{\vec{\nabla}_{\mathbf{r}_{i}}-\overleftarrow{\nabla}_{\mathbf{r}_{i}}}{2 m_{p} i} e^{i \boldsymbol{q} \cdot \boldsymbol{r}_{i}}+\sum_{i}^{A}\left(\nabla_{\boldsymbol{r}_{i}} \times \boldsymbol{\mu}\right) e^{i \boldsymbol{q} \cdot \boldsymbol{r}_{i}}
$$


are employed with the nonrelativistic RPA calculation. The spin-current operator in the second term of $\hat{J}_{N}(q)$ commutes with $\hat{\rho}_{N}$. Hence the spin current introduces no gauge violation into the NP calculation of Eq. A1). The convection-current, on the other hand, does not commute with $\hat{\rho}_{N}$ leading to a violation of gauge invariance:

$$
<I\left|\left[\hat{\rho}_{N}(-\boldsymbol{q}), \hat{j}_{N}^{\nu}\left(\boldsymbol{q}^{\prime}\right)\right]\right| I>=\frac{\boldsymbol{q}}{m_{p}} \rho_{N}\left(\boldsymbol{q}-\boldsymbol{q}^{\prime}\right)_{I I}
$$

Therefore the NP contribution given by Eq. (A1) is not gauge invariant with the impulse charge-current operators. When the seagull tensor of Eq. (6) is added to the nuclear polarization tensor of Eq. A3), this term is just canceled. Hence the gauge invariance of NP calculation is restored by the seagull term together with the the ladder and cross terms. 
[1] H. F. Beyer, G. Menzel, D. Liesen, A. Gallus, F. Bosch, R. Deslattes, P. Indelicato, Th. Stöhlker, O. Klepper, R. Moshammer, F. Nolden, H. Eickhoff, B. Franzke, and M.Steck, Z. Phys. D: At., Mol. Clusters 35, 169 (1995).

[2] J. R. Sapirstein and D. R. Yennie, in Quantum Electrodynamics, edited by T. Kinoshita (World Scientific, Singapore 1990), p. 560 .

[3] P. J. Mohr, G. Plunien and G. Soff, Phys. Rep. 293, 227 (1998).

[4] E. Borie and G. A. Rinker, Rev. Mod. Phys. 54, 67 (1982).

[5] B. Hoffmann, G. Baur, and J. Speth, Z. Phys. A - Atoms and Nuclei 315, 57 (1984); 320, 259 (1985).

[6] G. Plunien, B. Müller, W. Greiner, and G. Soff, Phys. Rev. A 39, 5428 (1989); 43, 5853 (1991).

[7] G. Plunien and G. Soff, Phys. Rev. A 51, 1119 (1995); 53, 4614(E) (1996).

[8] A. V. Nefiodov, L. N. Labzowsky, G. Plunien, and G. Soff, Phys. Lett. A 222, 227 (1996).

[9] R. K. Cole, Jr. , Phys. Rev. 177, 164 (1969).

[10] Y. Tanaka and Y. Horikawa, Nucl. Phys. A 580, 291 (1994).

[11] N. Yamanaka and A. Ichimura, Phys. Scr. bf T80, 488 (1999).

[12] N. Yamanaka, A. Haga, Y. Horikawa, and A. Ichimura, Phys. Rev. A 63, 062502 (2001).

[13] J. L. Friar and M. Rosen, Ann. Phys. (N.Y.) 87, 289 (1974).

[14] R. Rosenfelder, Nucl. Phys. A 393, 301 (1983).

[15] A. B. Migdal, Theory of finite Fermi systems and applications to atomic nuclei (Interscience, New York, 1967).

[16] A. Bohr and B. R. Mottelson, Nuclear Structure (Benjamin, New York, 1975), Vol. 2, p. 399.

[17] Y. Horikawa and Y. Tanaka, Phys. Lett. B 409, 1 (1997). 
TABLE I. The energy-weighted sums of $B(E \lambda)$ over the RPA states. The classical EWSR values [16] are also shown for comparison. The values are given in units of $\mathrm{e}^{2} \mathrm{~b}^{\lambda} \cdot \mathrm{MeV}$

\begin{tabular}{ccc}
\hline \hline $\mathrm{E} \lambda$ & Present calculation & Classical EWSR $^{a}$ \\
\hline$E 0^{b}$ & 1.97 & 1.64 \\
$E 1^{c}$ & 8.15 & 7.38 \\
$E 2$ & 22.2 & 20.5 \\
$E 3$ & 24.4 & 23.4 \\
$E 4$ & 14.2 & 23.6 \\
$E 5$ & 11.3 & 23.1 \\
$M 1^{d}$ & 294 & \\
\hline \hline
\end{tabular}

${ }^{a}$ The radial moments $\left\langle r^{\lambda}>_{p}\right.$ in the classical EWSR are calculated by the Fermi charge distribution (35).

${ }^{b}$ The $E 0$ operator is defined as $O(E 0)=\sum_{p} r^{2} / \sqrt{4 \pi}$.

${ }^{c}$ The $E 1$ operator is defined as $O(E 1)=\sum_{i}-1 / 2 \tau_{3} r Y_{1 \mu}$.

$d$ The value given in units of $\mu_{\mathrm{N}} \cdot \mathrm{MeV}$. 
TABLE II. Nuclear polarization correction $(\mathrm{meV})$ of the $1 s_{1 / 2}$ state in ${ }_{82}^{208} \mathrm{~Pb}^{81+}$. Energy shifts $\Delta E^{L}$, $\Delta E^{X}$ and $\Delta E^{S G}$ are contributions of the ladder, cross and seagull terms, respectively, while $\Delta E^{+}\left(\Delta E^{-}\right)$ denotes contribution from the positive (negative) energy intermediate states of the electron.

\begin{tabular}{|c|c|c|c|c|c|c|c|}
\hline$\lambda^{\pi}$ & Contribution & $\begin{array}{r}\text { present }^{a} \\
\text { Feynman(NP) } \\
\end{array}$ & $\begin{array}{r}\text { present }^{b} \\
\text { Coulomb(NP) }\end{array}$ & $\begin{array}{r}\text { present }^{c} \\
\text { CNP }\end{array}$ & Ref. $\frac{[12]^{d}}{\mathrm{NP}}$ & Ref. ${ }_{\mathrm{CNP}}^{12}{ }^{e}$ & Ref. $\left[\frac{8]^{f}}{\mathrm{CNP}}\right.$ \\
\hline $0^{+}$ & $\begin{array}{l}\Delta E^{L+} \\
\Delta E^{L-} \\
\Delta E^{X+} \\
\Delta E^{X-} \\
\Delta E^{L}+\Delta E^{X} \\
\Delta E^{S G+} \\
\Delta E^{S G-} \\
\Delta E^{L}+\Delta E^{X}+\Delta E^{S G}\end{array}$ & $\begin{array}{l}-5.7 \\
+0.4 \\
-1.2 \\
+2.7 \\
-3.8 \\
+0.7 \\
-0.9 \\
-3.9\end{array}$ & $\begin{array}{r}-6.5 \\
+0.2 \\
-0.2 \\
+2.7 \\
-3.9 \\
0.0 \\
0.0 \\
-3.9\end{array}$ & $\begin{array}{l}+3.0 \\
-4.0\end{array}$ & -6.6 & -7.2 & -3.3 \\
\hline $1^{-}$ & $\begin{array}{l}\Delta E^{L+} \\
\Delta E^{L-} \\
\Delta E^{X+} \\
\Delta E^{X-} \\
\Delta E^{L}+\Delta E^{X} \\
\Delta E^{S G+} \\
\Delta E^{S G-} \\
\Delta E^{L}+\Delta E^{X}+\Delta E^{S G}\end{array}$ & $\begin{array}{r}-119.1 \\
+74.0 \\
-49.8 \\
+110.1 \\
+15.2 \\
+144.2 \\
-186.5 \\
-27.1\end{array}$ & $\begin{array}{l}-91.1 \\
+37.6 \\
-29.4 \\
+64.2 \\
-18.7 \\
+87.8 \\
-95.1 \\
-26.0\end{array}$ & $\begin{array}{l} \\
+16.7 \\
-20.3\end{array}$ & +16.3 & -19.5 & -17.6 \\
\hline $2^{+}$ & $\begin{array}{l}\Delta E^{L+} \\
\Delta E^{L-} \\
\Delta E^{X+} \\
\Delta E^{X-} \\
\Delta E^{L}+\Delta E^{X} \\
\Delta E^{S G+} \\
\Delta E^{S G-} \\
\Delta E^{L}+\Delta E^{X}+\Delta E^{S G}\end{array}$ & $\begin{array}{r}-13.0 \\
+2.0 \\
-3.2 \\
+8.1 \\
-6.1 \\
+4.4 \\
-4.1 \\
-5.7\end{array}$ & $\begin{array}{r}-15.3 \\
+0.4 \\
-0.5 \\
+9.1 \\
-6.3 \\
+2.7 \\
-2.1 \\
-5.7\end{array}$ & $\begin{array}{l}+8.6 \\
-5.8\end{array}$ & -7.0 & -6.3 & -5.8 \\
\hline $3^{-}$ & $\begin{array}{l}\Delta E^{L+} \\
\Delta E^{L-} \\
\Delta E^{X+} \\
\Delta E^{X-} \\
\Delta E^{L}+\Delta E^{X} \\
\Delta E^{S G+} \\
\Delta E^{S G-} \\
\Delta E^{L}+\Delta E^{X}+\Delta E^{S G}\end{array}$ & $\begin{array}{l}-5.0 \\
+1.0 \\
-1.6 \\
+3.2 \\
-2.4 \\
+1.0 \\
-0.8 \\
-2.2\end{array}$ & $\begin{array}{l}-6.5 \\
+0.1 \\
-0.1 \\
+4.1 \\
-2.4 \\
+0.6 \\
-0.4 \\
-2.2\end{array}$ & $\begin{array}{l}+4.0 \\
-2.3\end{array}$ & -2.9 & -2.6 & -2.6 \\
\hline $4^{+}$ & $\begin{array}{l}\Delta E^{L+} \\
\Delta E^{L-} \\
\Delta E^{X+} \\
\Delta E^{X-} \\
\Delta E^{L}+\Delta E^{X} \\
\Delta E^{S G+} \\
\Delta E^{S G-} \\
\Delta E^{L}+\Delta E^{X}+\Delta E^{S G}\end{array}$ & $\begin{array}{l}-1.1 \\
+0.2 \\
-0.4 \\
+0.7 \\
-0.7 \\
+0.5 \\
-0.4 \\
-0.6\end{array}$ & $\begin{array}{r}-1.5 \\
0.0 \\
0.0 \\
+0.8 \\
-0.7 \\
+0.4 \\
-0.3 \\
-0.6\end{array}$ & $\begin{array}{l}+0.8 \\
-0.6\end{array}$ & & & \\
\hline
\end{tabular}


TABLE II. (Continued).

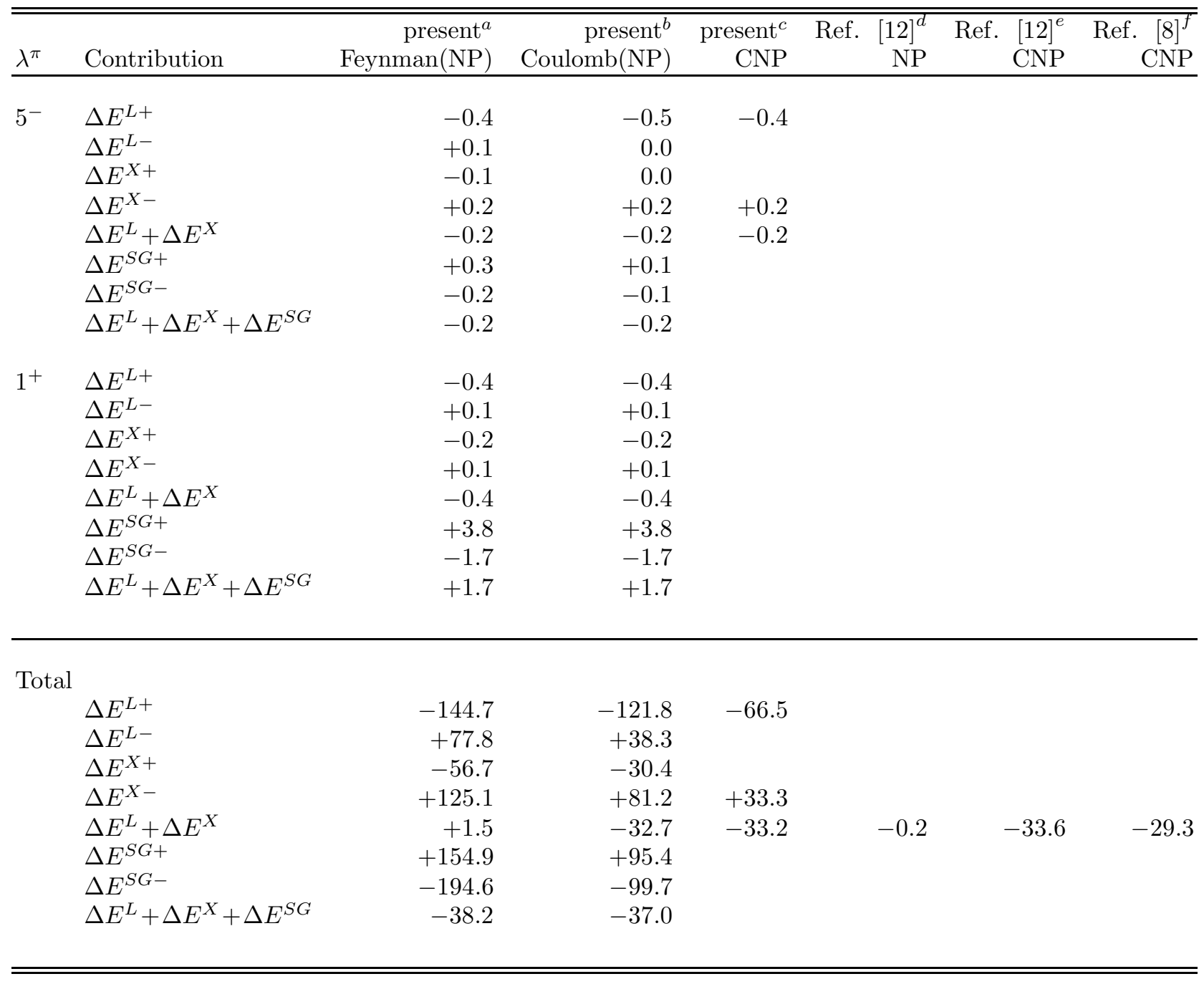

${ }^{a}$ The NP energies in the Feynman gauge.

${ }^{b}$ The NP energies in the Coulomb gauge.

${ }^{c}$ The unretarded NP energies in the Coulomb gauge.

${ }^{d}$ The NP energies evaluated in the Feynman gauge. Electron wave functions were solved by assuming the point charge for the nuclear ground state. Nuclear transition-charge densities were determined by a collective model. They were normalized to the observed $B(E \lambda)$ for the low-lying nuclear states and EWSR values for the high-lying giant resonances. Nuclear current densities $J_{N \lambda \lambda-1}(r)$ were obtained by solving the equation of the charge conservation (42) assuming $J_{N \lambda \lambda+1}(r)=0$.

$e$ Same as $d$ except for the unretarded NP energies.

$f$ The unretarded NP energies. Same as $e$ except for the electron wave functions solved by assuming a finite charge distribution for the nuclear ground state. 
TABLE III. Total nuclear polarization (meV) of the $1 s_{1 / 2}, 2 s_{1 / 2}, 2 p_{1 / 2}$, and $2 p_{3 / 2}$ states of ${ }_{82}^{208} \mathrm{~Pb}^{81+}$ both in the Feynman and Coulomb gauges. The abbreviation CNP denotes the unretarded results.

\begin{tabular}{lccc}
\hline \hline States & Feynman(NP) & Coulomb(NP) & CNP \\
\hline $1 \mathrm{~s}_{1 / 2}$ & -38.2 & -37.0 & -33.2 \\
$2 \mathrm{~s}_{1 / 2}$ & -6.7 & -6.4 & -5.7 \\
$2 \mathrm{p}_{1 / 2}$ & -0.2 & -0.2 & -0.6 \\
$2 \mathrm{p}_{3 / 2}$ & +0.0 & +0.0 & -0.0 \\
\hline \hline
\end{tabular}




\section{Figure captions}

FIG. 1. Diagrams contributing to nuclear polarization in lowest order; (a) ladder diagram, (b) cross diagram, (c) seagull diagram.

FIG. 2. Electronic Coulomb form-factors $<E_{i^{\prime}}, p_{1 / 2}\left\|m_{1}(q)\right\| 1 s_{1 / 2}>$ for the $E_{i^{\prime}}=2,6$ and $10 \mathrm{MeV}$ states (solid line). The dotted line is the nuclear Coulomb form factor for the $14.6 \mathrm{MeV}^{-}$state.

FIG. 3. Nuclear polarization spectra as functions of nuclear excitation energy. The Coulomb gauge was assumed.

FIG. 4. The transition potentials due to the nuclear $1^{-}, 14.6 \mathrm{MeV}$ state. The Coulomb-Breit propagator was assumed. The solid, dotted and dash-dotted lines denote the potentials corresponding to $\rho_{1}(\mathrm{r})$,

$J_{10}^{T}(\mathrm{r})$ and $J_{12}^{T}(\mathrm{r})$, respectively. The transverse potential $J_{10}^{T}(\mathrm{r})$ is seen to be larger than the Coulomb potential in the region $r>60 \mathrm{fm}$.

FIG. 5. Spectral densities of nuclear polarization for (a) $0^{+}, 13.3 \mathrm{MeV}$, (b) $1^{-}, 14.6 \mathrm{MeV}$ and (c) $2^{+}, 10.2 \mathrm{MeV}$ states as functions of electron energy $E_{i^{\prime}}$. The solid line denotes calculation with both the Coulomb and transverse parts of the electromagnetic interaction, while the dotted line denotes calculation with only the Coulomb part of the interaction. Electron intermediate states, (a) $\mid E_{i^{\prime}}, s_{1 / 2}>$, (b) $\mid E_{i^{\prime}}, p_{1 / 2}>$ and (c) $\mid E_{i^{\prime}}, d_{3 / 2}>$ are assumed in the respective panels. 